\title{
Potential role of mitochondria in gastric cancer detection: Fission and glycolysis (Review)
}

\author{
HANG YANG, YAN LI and BING HU \\ Department of Gastroenterology, West China Hospital, Sichuan University, Chengdu, Sichuan 610041, P.R. China
}

Received January 10, 2021; Accepted March 22, 2021

DOI: 10.3892/ol.2021.12700

\begin{abstract}
Gastric cancer (GC) is characterized by high morbidity and mortality rates worldwide. Helicobacter pylori infection, high salt intake, smoking, alcohol, low fiber intake, family history of GC, obesity and precancerous lesions, including chronic atrophic gastritis and intestinal metaplasia, are considered general risk factors for GC. Image enhancement endoscopy methods, which improve the visualization of mucosal structures and vascularity, may be used for the early diagnosis of GC, such as narrow band imaging, which can reveal fine details of subtle superficial abnormalities of early gastric cancer (EGC). Mitochondria are well-known for their role in producing ATP via the tricarboxylic acid cycle. In cancer cells, the energetic metabolism can be reprogrammed as anaerobic glycolysis for energy production and anabolic growth. In addition to their dominant metabolic functions, mitochondria participate in several central signaling pathways, such as the apoptotic pathway and NLRP3 inflammasome activation. Conversely, mitochondrial dynamics, including fission/fusion and mitophagy, can also contribute to the pathogenesis of cancer. The dysfunction and dysregulation of mitochondria have been associated with several ageing and degenerative diseases, as well as cancer. The present review focuses on energy metabolism and mitochondrial dynamics, and summarizes the changes in gastric carcinogenesis, the diagnosis of EGC and indicates potential targeted treatments.
\end{abstract}

\section{Contents}

1. Introduction

2. Mitochondrial dynamics: Fission and fusion

3. Fission, mitophagy and GC

4. Fusion and GC

Correspondence to: Professor Bing $\mathrm{Hu}$, Department of Gastroenterology, West China Hospital, Sichuan University, 37 Guo Xue Xiang, Wu Hou, Chengdu, Sichuan 610041, P.R. China E-mail: hubingnj@163.com

Key words: diagnosis of early gastric cancer, mitochondrial dynamics, reprogrammed energy metabolism
5. Reprogrammed energy metabolism and GC

6. Association between mitochondrial dynamics and energy metabolism: Fission and glycolysis

7. Conclusions

\section{Introduction}

Chronic atrophic gastritis (CAG) is considered a common risk factor for the development of gastric cancer (GC). Endoscopic imaging and biopsy are crucial for early detection and diagnosis of GC (1). Image-enhanced endoscopy combined with biopsy, according to the Sydney protocol and regular endoscopic surveillance, are recommended for patients with extensive CAG or intestinal metaplasia (2). A visible lesion may be treated by endoscopic mucosal resection or endoscopic submucosal dissection. However, when a lesion is invisible, regular endoscopic surveillance is required for high-risk patients. The interval between Helicobacter pylori eradication and cancer occurrence may vary from several months to $>10$ years (3). Surveillance endoscopy is one of the methods enabling the early diagnosis of GC (4). Once an existing lesion is identified, it can be treated in a timely manner. Interval cancer can occur due to missed lesions or to a newly developed lesion during surveillance (5). Thus, it is essential to identify a molecular biological marker for the detection of invisible lesions at the organelle level.

Recently, diverse pathophysiological functions of mitochondria have been reported, including mitochondrial dynamics (6), metabolic reprogramming (7), mitochondria-released damage-associated molecular patterns and NLRP3 inflammasome activation (8), mitochondrial DNA (mtDNA), autophagy and mitophagy (9), mitochondrial outer membrane permeabilization (10) and mitochondrial aging (11). In addition, mitochondrial (mt)DNA mutations, deletions and impaired DNA replication are the most common causes of mitochondrial dysfunction (12). mtDNA sensing via STING signaling participates in inflammation and cancer $(12,13)$. The effects of mitochondrial dynamics on carcinogenesis and cancer progression have also been reported, highlighting the potential use of mitochondrial biomarkers in cancer detection and prognosis, as well as the potential targeting of mitochondrial dynamics for treating cancer (14). However, there is still a paucity of research associated with GC. 
The present review summarizes the role of mitochondrial dynamics and energy metabolism reprogramming in GC to identify potential indicators for biologically complemented endoscopy and further promote translating discoveries of molecular biology. Thus, fission and glycolysis from mitochondria may be useful in detecting GC. If an electron microscope can be installed on the endoscopy system, the mitochondrial dynamics may be observable during the early stages of GC. Furthermore, when fission is increased and fusion is decreased, further precision biopsy of the targeted tissue should be performed to detect metabolic activity. The combination of both approaches may enable early diagnosis and provide a novel treatment strategy. However, further investigation is required.

\section{Mitochondrial dynamics: Fission and fusion}

Mitochondria are responsible for energy supply and are involved in several biological processes, including cell death and proliferation (6). Mitochondria constantly maintain a dynamic shape, which may change in response to cellular bioenergetic demands, such as nutrient status, which is defined as mitochondrial dynamics (12). The mitochondrial morphology is a result of the interplay between rapid fusion and fission events (15). The key components mediating these processes belong to the dynamin family of GTPases that utilize GTP hydrolysis to drive mechanical work on biological membranes (16). Mitofusin proteins, Mfn1 and Mfn2, are involved in the fusion of the outer mitochondrial membrane, while GTPase optic atrophy 1 mediates the fusion of the inner mitochondrial membrane (17). Mitochondrial fission is mediated by the GTPase dynamin-related protein 1 (Drp1) following its recruitment by the membrane-anchored proteins, namely mitochondrial fission factor and fission protein 1 (Fis1) (18). Commonly, the mitochondrial fission/fusion machinery is involved in generating new mitochondria, and eliminating old, damaged and non-repairable mitochondria (6). Mitochondrial fission plays an important role in mitochondrial proliferation, mitochondrial distribution during cell division and the removal of damaged mitochondria via mitophagy (19). Unopposed mitochondrial fission causes mitochondrial fragmentation, which is generally associated with metabolic dysfunction and several diseases, such as degenerative diseases and cancer (20). It has been reported that impaired mitochondrial fission is associated with mitochondrial elongation (21). In addition, unopposed fusion results in a hyperfused network and serves to counteract metabolic insults, preserve cellular integrity and protect against autophagy (20). It was previously reported that impaired mitochondrial fusion may promote fission-induced mitochondrial fragmentation (21). Thus, the maintenance of mitochondrial fission/fusion balance plays a key role in cell cycle progression (6). The dynamics is critical for the effects of fission/fusion on morphology regulation, content exchange, and the maintenance of mtDNA and mitochondrial oxidative phosphorylation (OXPHOS) activity $(22,23)$.

\section{Fission, mitophagy and GC}

Fission isolates depolarized mitochondria, while it coordinates the downregulation of fusion mediators to prevent network reintegration, thereby facilitating mitophagy, mainly via interactions between Parkin, Bcl-2/adenovirus E1B $19 \mathrm{kDa}$ protein interacting protein 3 (BNIP3) and Drp1 (24). Increasing Drp1 results in excessive mitochondrial fragmentation and deficiencies, decreases mitochondrial motility and shortens mitochondrial length (25), which may be further enhanced in hypoxia (26). Fission can also be triggered by stress stimuli, such as nutrient deprivation, DNA damage, inflammation and mitochondrial membrane depolarization (27). Given that mitochondria-associated membranes related to the endoplasmic reticulum at specific regions can facilitate calcium $\left(\mathrm{Ca}^{2+}\right)$ flux into the mitochondria and further control the homeostasis and metabolism of $\mathrm{Ca}^{2+}$, close coupling of these organelles increases mitochondrial $\mathrm{Ca}^{2+}$ levels, thus initiating apoptosis (28). It has also been reported that enhanced fission attenuates adherence to inhibit $\mathrm{Ca}^{2+}$ overload in mitochondria and apoptosis (29). In terms of mitophagy, this process maintains cellular health by selectively enclosing damaged and depolarized mitochondria in autophagic vacuoles for lysosome-mediated elimination (30). Mitophagy degrades dysfunctional mitochondria and further attenuates reactive oxygen species (ROS) generation, which in turn promotes cell survival and protects against cell death (31). Increasing evidence suggest that several modulators of mitophagy are deregulated in human cancer, including Parkinson protein 2 E3 ubiquitin protein ligase, FUN14 domain containing 1, BNIP3 and BNIP3L $(32,33)$. In addition, a study revealed that impaired mitophagy can enhance the aggressiveness in GC cells under hypoxia by activating the mtROS/hypoxia-inducible factor (HIF)- $1 \alpha$ interplay (34). Mitophagy may also be enhanced by overexpression of Opa-interacting protein 5, thus plays an important role in cell survival and death in docetaxel-treated GC cells (35). Another study demonstrated that Drp1 expression is upregulated, and the expression levels of the mitophagy-related regulators, PTEN-induced putative kinase 1 and Parkin, are downregulated in patients with GC (36). Given that mitophagy can clear the damaged part of mitochondria and mtDNA, it protects healthy cells from malignant transformation and tumor cells from apoptosis (31). It has been suggested that, in the early stages of GC, mitophagy is associated with tumor suppression, whereby it can promote tumor growth at the advanced stages of GC. For example, mitophagy was increased in advanced-stage GC to sustain the viability and migration of GC cells (37), since mitophagy in solids tumor may be activated by two common factors, namely hypoxia and low nutrient supply (38) (Fig. 1).

\section{Fusion and GC}

Mitochondrial fusion results in a more interconnected mitochondrial network and enhances the communication with the endoplasmic reticulum (39). Fusion allows the diffusion of matrix content among mitochondria, diluting the accumulated mtDNA mutations and oxidized proteins (40). Fusion is commonly enhanced by starvation by triggering the protein kinase A-mediated phosphorylation of Drp1 (at Ser637) to blunt fission (41). In addition, mitochondrial fusion is required for mtDNA maintenance (22). Thus, impaired mitochondrial fusion is often accompanied by bioenergetic defects due to loss of mtDNA (42). Furthermore, mitochondrial fusion is also associated with increased OXPHOS and ATP generation 


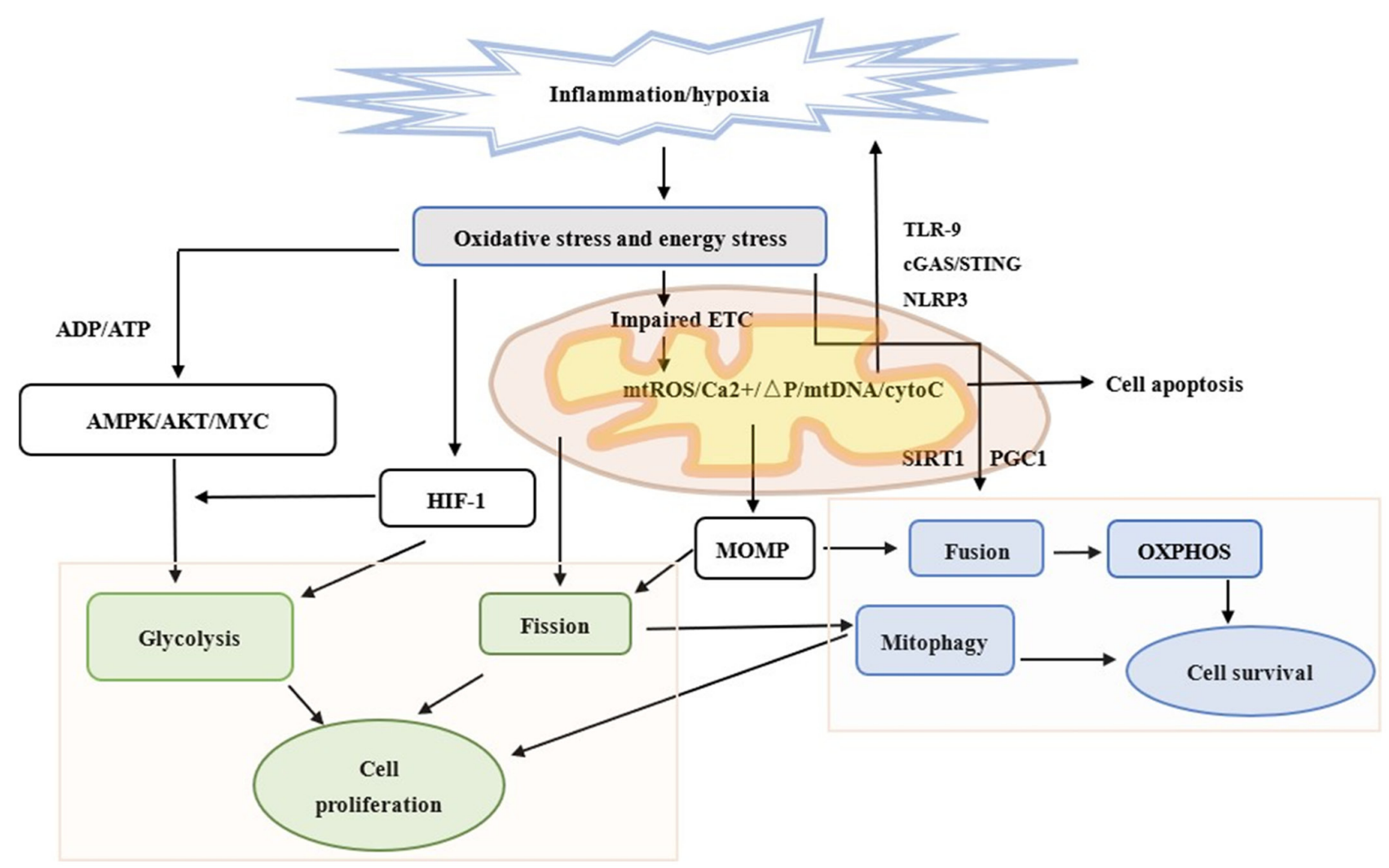

Figure 1. Different mitochondrial dynamics and energy metabolism in an epithelial cell subjected to chronic inflammation. Chronic inflammation causes the injury of epithelial cells. Mitochondria is involved in further innate immune responses, including cGAS-STING signaling, TLR-9 and NLRP3 inflammasome formation following the release of mtDNA. Mitochondria is also associated with apoptosis. When atrophic epithelial cells preserve their programed cell death ability and surrounding inflammation is sufficiently severe, cells undergo apoptosis instead of necrosis. Chronic inflammation can also damage mitochondria and lead to changes in mitochondrial metabolism and dynamics via HIF-1, AMPK and MOMP. Fission and glycolysis promote cell proliferation and invasion. Fusion and OXPHOS are compatible with cell survival. Mitophagy protects both normal and cancer cells by selectively eliminating damaged mitochondria. Green outline represents proliferation, blue outline represents survival and the text without boxes represent apoptosis. cGAS, cyclic GMP-AMP synthase; STING, stimulator of interferon genes; TLR-9, Toll-like receptor-9; NLRP3, NOD-like receptor family pyrin domain-containing 3; mtDNA, mitochondrial DNA; HIF-1, hypoxia-inducible factor-1; AMPK, AMP-activated protein kinase; MOMP, mitochondrial outer membrane permeabilization; OXPHOS, oxidative phosphorylation; ETC, electron transport chain; mtROS, mitochondrial ROS; $\Delta \mathrm{P}$, increased potential; cytoC, cytochrome c; PGC1, proliferator-activated receptor- $\gamma$ coactivator.

via remodeling of the cristae $(43,44)$, and downregulation of OPA1, which is responsible for fusion, resulted in mitochondrial dysfunction and mtDNA stress (45). The number of mitochondria is regulated by mitochondrial biogenesis to meet the energy demands of the cells and compensate for their damage (46). A study demonstrated that peroxisome proliferator-activated receptor gamma coactivator (PGC-1) and the protein deacetylase sirtuin 1 (SIRT1) can regulate fusion and OXPHOS (14). Thus, activation of PGC-1 $\alpha$ by SIRT1 induces mitochondrial biogenesis and confers metabolic advantages (14). Another study revealed that PGC-1 $\beta$ can induce mitochondrial fusion by upregulating Mfn2 expression via estrogen-related receptor $\alpha$ coactivation (47). Mfn2 expression is downregulated in GC tissues compared with normal gastric mucosal tissues, and is negatively associated with tumor size, indicating an antitumor role of Mfn2 (48). In vitro experiments have demonstrated that overexpression of Mfn2 can suppress gastric cancer cell proliferation and colony formation (48). SIRT1 is an enzyme that mediates NAD ${ }^{+}$-dependent deacetylation of target substrates (49). Given that the cellular redox balance of $\mathrm{NAD}^{+}$and $\mathrm{NADH}$ is highly associated with catabolic fluxes, SIRT1 can act as a sensor, directly connecting metabolic perturbations with transcriptional output (49). SIRT1 expression is significantly downregulated in GC tissues, which is associated with poor prognosis (50). It has also been reported that SIRT1 exerts inhibitory effects on chemoresistance and cancer stem cell properties via Forkhead box $\mathrm{O} 3$ and AMP-activated protein kinase (AMPK) (51). AMPK, another key energy metabolic sensor, plays a key role in maintaining cellular energy homeostasis and is activated upon alterations in the cellular AMP/ATP ratio (52). Previous studies have demonstrated that, upon energy deficiency, AMPK activation may result in increased PGC-1 $\alpha$ expression and phosphorylation to modulate the expression of several key players in mitochondrial biogenesis and OXPHOS of fatty acids $(53,54)$ (Fig. 1).

\section{Reprogrammed energy metabolism and GC}

Energy metabolism is essential for maintaining cellular homeostasis and biological functions, and includes ATP production in the cytosol (glycolysis) and mitochondria (OXPHOS) (55), which can be reprogrammed during carcinogenesis (56). Cancer cells undergo metabolic reprogramming, including enhanced glycolysis, mutations in genes encoding tricarboxylic acid (TCA) cycle enzymes, upregulation of de novo lipid synthesis and glutaminolysis (57). Glycolysis is characterized by an increased rate of glucose uptake and its glycolytic conversion to lactate, even under oxygen-rich conditions (55). There 
are several pathways and transcriptional regulators involved in the regulation of metabolic reprogramming, such as PI3K/AKT pathway and HIF-1 $(58,59)$. The PI3K/AKT pathway can regulate several aspects of this metabolic program (58). A previous study demonstrated that AKT activation was sufficient to induce glycolysis by promoting glucose transporter 1 and phosphorylating pyruvate dehydrogenase kinase to inhibit pyruvate dehydrogenase and favor lactate dehydrogenase (LDH) activity (60). It has been reported that HIF-1 is overexpressed in human cancers as a result of intratumoral hypoxia, as well as genetic alterations, such as gain-of-function mutations in oncogenes and loss-of-function mutations in tumor suppressor genes (61). HIF-1 may also be triggered by the accumulation of TCA substrates (62), while its degradation is regulated by $\mathrm{O}_{2}$-dependent prolyl hydroxylation (PHs) (61). HIF-1 $\alpha$ maintains its stability by avoiding the hydroxylation of PHs in cancer cells, since PHs can be inhibited by the increased levels of cytosolic pyruvate, lactate, succinate, fumarate and ROS (59). Most genes encoding glycolytic enzymes and transporters are the targets of HIF-1 $\alpha$, and its overexpression in cancer cells is associated with increased levels of glycolytic proteins (63). A study revealed that HIF-1 $\alpha$ levels were high in certain tumors, even under oxygen-rich conditions, indicating that hormones or growth factors can cause the stabilization of HIF-1 $\alpha$ expression, which may serve important roles in carcinogenesis (64). A previous study suggested that HIF- $1 \alpha$ can act as a negative regulator of mitochondrial biogenesis and oxidative phosphorylation to inhibit the conversion of pyruvate to acetyl-CoA and mitochondrial respiration and to promote LDH expression (65). HIF-1 $\alpha$ activation can also inhibit MYC transcription to further downregulate PGC- $1 \alpha$ and PGC-1 $\beta$ expression, which in turn regulates mitochondrial biogenesis and OXPHOS (54). In GC, inhibiting HIF-1 $\alpha$ signaling attenuates the migratory and invasive abilities of GC cells, and epithelial-to-mesenchymal transition (66), whereas activation of HIF-1 $\alpha$ signaling promotes cell metastasis and glucose metabolism (67).

The tumor microenvironment favors the growth and expansion of cancer and inflammatory cells, which in turn directly or indirectly promotes gastric tumorigenesis by secreting soluble factors or modulating immune responses (68). It has been reported that NF- $\kappa \mathrm{B}$ is activated in chronic inflammation, thus promoting the further activation of tumor-promoting genes, such as IL-6 and cyclooxygenase (COX)-2 (69). NF- $\mathrm{BB}$ and HIF-1 can link inflammatory signaling to hypoxia and coordinate the activation of both COX-2 and IL-6, and the Janus kinase/STAT3 pathway (70). It has been reported that STAT3 cooperates with NF- $\kappa$ B and HIF-1 in the regulation of both genes (71). NF- $\kappa \mathrm{B}$ can be strongly induced by hypoxia and chronic inflammation, and is involved in the reprogramming of tumor glycolysis by interacting with HIF-1 $\alpha$ (70). Given that inflammation can induce cells lacking oxygen and upregulate HIF-1 $\alpha$, glycolysis gradually becomes the main energy source instead of OXPHOS (55) (Fig. 1)

\section{Association between mitochondrial dynamics and en- ergy metabolism: Fission and glycolysis}

Mitochondrial morphological changes are a type of primary signal to shape metabolic reprogramming during cellular quiescence or activation $(14,72)$. Recent studies have demonstrated that increased mitochondrial fission promotes a pro-tumorigenic phenotype $(12,73,74)$. Several studies have been performed in different cell types that alter their mitochondrial morphology to meet their energy demands, functions and behaviors. Conversely, certain cells, such as T cells and stem cells, have higher energy demands to perform their metabolic and cell-specific functions (75,76). When $\mathrm{T}$ cells recognize major histocompatibility complexes presented by antigen-presenting cells in response to infection or tumors, they proliferate and differentiate into different T-cell subsets (23). Effector T cells display looser cristae remodeling via fission with reduced electron transport chain (ETC) complexes, thus attenuating ETC efficiency and promoting aerobic glycolysis (23). Conversely, in memory $\mathrm{T}$ cells, tight cristae remodeling via fusion with enhanced ETC complex activity is observed, thus enhancing ETC efficiency and OXPHOS (23). Endothelial progenitor cells (EPCs) accelerate glycolysis to produce lactate during angiogenesis by upregulating the expression levels of HIF $\square 1 \alpha$ and vascular endothelial growth factor (77). In human EPCs, downregulation of Fis1 expression is associated with mitochondrial dysfunction and may contribute to the impaired activity of EPCs during the senescence process (73). However, upregulation of Fis1 expression in senescent EPCs restores the younger phenotype (73). Another study investigated the function of mitochondrial fission genes in embryonic stem cells (ESCs). Transmission electron microscopy revealed a significant increase in the cytoplasm-to-nucleus ratio and mitochondrial elongation in dynamin-1-like protein (-/-) ESCs caused by incomplete fission. In addition, increased OXPHOS and intracellular ATP concentration and reduced glycolysis was observed, which were associated with mitochondrial elongation (78). The proliferation and invasion of tumor cells also require faster and increased energy supply (79). Thus, Drp1 expression is upregulated in several types of cancer cells, including liver (80), breast (81) and lung cancers (82), and may be considered as a biomarker for predicting poor survival in patients with these types of cancer. A study on ovarian cancer demonstrated that glycolysis is promoted by activating PI3K/AKT/HIF-1 $\alpha$ signaling, while mitochondrial fission is enhanced by phosphorylation of Drp1 at Ser616 (83). As a member of the AMPK family, salt-inducible kinase 2 was demonstrated to be involved in both pathways (83). In addition, Drp1 expression was significantly upregulated in pancreatic cancer (PC) cells and tissues via downregulation of microRNA-29a expression (74). High Drp1 expression was associated with poor survival of patients with PC, while Drpl promoted both the proliferation and metastasis of PC cells, mainly through facilitating aerobic glycolysis (74). Another study revealed that Drp1 may promote KRAS-driven tumor growth by supporting both glycolysis and mitochondrial function (84). Taken together, these findings suggest a mutual association between Drp1 and glycolysis, and the promoting effect of Drp1 and glycolysis on cancer cell proliferation and invasion.

\section{Conclusions}

GC is the fifth most common type of cancer and the third most common cause of cancer-associated mortality, with 784,000 mortalities reported in 2018 worldwide (85). Early detection and treatment can improve the outcome of patients with GC. Innovative endoscopic techniques may be more accurate in achieving cytological or even biological diagnosis. Mitochondria 
are strongly associated with carcinogenesis. The present review summarized the role of mitochondria dynamics, reprogramming of energy metabolism and their changes in GC. Based on current literature, it can be concluded that mitochondria in GC are characterized by fission and enhanced glycolysis to meet the increased energy requirements of cancer cells, and decrease necrosis via mitophagy. Upregulated expression levels of Drp1 and HIF-1 $\alpha$ are associated with fission and glycolysis, respectively. The balance of mitochondrial fission and fusion and the ratio of glycolysis to OXPHOS are positively associated with different stages of carcinogenesis. When increased fission and glycolysis and decreased apoptosis and fusion are detected in high-risk patients, they may indicate that cells are in the process of malignant transformation. Thus, treatment is required to inhibit this process, which may be a promising approach to the detection of early gastric cancer via organelle- and molecular-level endoscopy in the future.

\section{Acknowledgements}

Not applicable.

\section{Funding}

The present study was supported by the 1.3 .5 project for disciplines of excellence Clinical Research Incubation Project, West China Hospital, Sichuan University, China (grant no. 20HXFH016).

\section{Availability of data and materials}

Not applicable.

\section{Authors' contributions}

HY designed the present review and drafted the initial manuscript. $\mathrm{BH}$ contributed to designing and reviewing the manuscript. $\mathrm{HY}$, $\mathrm{YL}$ and $\mathrm{BH}$ contributed to revising the manuscript for important intellectual content. Data authentication is not applicable. All authors have read and approved the final manuscript.

\section{Ethics approval and consent to participate}

Not applicable.

\section{Patient consent for publication}

Not applicable.

\section{Competing interests}

The authors declare that they have no competing interests.

\section{References}

1. Pimentel-Nunes P, Libânio D, Marcos-Pinto R, Areia M, Leja M Esposito G, Garrido M, Kikuste I, Megraud F, MatysiakBudnik T, et al: Management of epithelial precancerous conditions and lesions in the stomach (MAPS II): European Society of Gastrointestinal Endoscopy (ESGE), European Helicobacter and Microbiota Study Group (EHMSG), European Society of Pathology (ESP), and Sociedade Portuguesa de Endoscopia Digestiva (SPED) guideline update 2019. Endoscopy 51: 365-388, 2019.
2. Banks M, Graham D, Jansen M, Gotoda T, Coda S, di Pietro M, Uedo N, Bhandari P, Pritchard DM, Kuipers EJ, et al: British Society of Gastroenterology guidelines on the diagnosis and management of patients at risk of gastric adenocarcinoma. Gut 68: 1545-1575, 2019.

3. Take S, Mizuno M, Ishiki K, Kusumoto C, Imada T, Hamada F, Yoshida T, Yokota K, Mitsuhashi T and Okada H: Risk of gastric cancer in the second decade of follow-up after Helicobacter pylori eradication. J Gastroenterol 55: 281-288, 2020.

4. Shichijo S and Hirata Y: Characteristics and predictors of gastric cancer after Helicobacter pylori eradication. World J Gastroenterol 24: 2163-2172, 2018.

5. Choi SI, Park B, Joo J, Kim YI, Lee JY, Kim CG, Choi IJ, Kook MC and Cho SJ: Three-year interval for endoscopic screening may reduce the mortality in patients with gastric cancer. Surg Endosc 33: 861-869, 2019.

6. Horbay R and Bilyy R: Mitochondrial dynamics during cell cycling. Apoptosis 21: 1327-1335, 2016.

7. Kim SY: Cancer Energy Metabolism: Shutting Power off Cancer Factory. Biomol Ther (Seoul) 26: 39-44, 2018.

8. Zhou R, Yazdi AS, Menu P and Tschopp J: A role for mitochondria in NLRP3 inflammasome activation. Nature 469: 221-225, 2011.

9. Jung S, Jeong $\mathrm{H}$ and $\mathrm{Yu} \mathrm{SW}$ : Autophagy as a decisive process for cell death. Exp Mol Med 52: 921-930, 2020.

10. Bock FJ and Tait SWG: Mitochondria as multifaceted regulators of cell death. Nat Rev Mol Cell Biol 21: 85-100, 2020.

11. Sun N, Youle RJ and Finkel T: The Mitochondrial Basis of Aging. Mol Cell 61: 654-666, 2016.

12. SrinivasanS, Guha M,Kashina A and Avadhani NG: Mitochondrial dysfunction and mitochondrial dynamics-The cancer connection. Biochim Biophys Acta Bioenerg 1858: 602-614, 2017.

13. Liu S, Feng M and Guan W: Mitochondrial DNA sensing by STING signaling participates in inflammation, cancer and beyond. Int J Cancer 139: 736-741, 2016.

14. Maycotte P, Marín-Hernández A, Goyri-Aguirre M, Anaya-Ruiz M, Reyes-Leyva J and Cortés-Hernández P: Mitochondrial dynamics and cancer. Tumour Biol: May 4, 2017 (Epub ahead of print). doi: 10.1177/1010428317698391.

15. Liu X, Weaver D, Shirihai O and Hajnóczky G: Mitochondrial 'kiss-and-run': Interplay between mitochondrial motility and fusion-fission dynamics. EMBO J 28: 3074-3089, 2009.

16. Chan DC: Mitochondrial dynamics and its involvement in disease. Annu Rev Pathol 15: 235-259, 2020.

17. Schrepfer E and Scorrano L: Mitofusins, from mitochondria to metabolism. Mol Cell 61: 683-694, 2016.

18. Cantó C: Mitochondrial dynamics: Shaping metabolic adaptation. Int Rev Cell Mol Biol 340: 129-167, 2018.

19. Ni HM, Williams JA and Ding WX: Mitochondrial dynamics and mitochondrial quality control. Redox Biol 4: 6-13, 2015.

20. Wai T and Langer T: Mitochondrial dynamics and metabolic regulation. Trends Endocrinol Metab 27: 105-117, 2016.

21. Bhatia D, Capili A and Choi ME: Mitochondrial dysfunction in kidney injury, inflammation, and disease: Potential therapeutic approaches. Kidney Res Clin Pract 39: 244-258, 2020.

22. Yan C, Duanmu X, Zeng L, Liu B and Song Z: Mitochondrial DNA: Distribution, mutations, and elimination. Cells 8: 379, 2019.

23. Buck MD, O'Sullivan D, Klein Geltink RI, Curtis JD, Chang CH, Sanin DE, Qiu J, Kretz O, Braas D, van der Windt GJ, et al: Mitochondrial dynamics controls $\mathrm{T}$ cell fate through metabolic programming. Cell 166: 63-76, 2016.

24. Friedman JR and Nunnari J: Mitochondrial form and function. Nature 505: 335-343, 2014.

25. Campello S, Lacalle RA, Bettella M, Mañes S, Scorrano L and Viola A: Orchestration of lymphocyte chemotaxis by mitochondrial dynamics. J Exp Med 203: 2879-2886, 2006.

26. Wu W, Li W, Chen H, Jiang L, Zhu R and Feng D: FUNDC1 is a novel mitochondrial-associated-membrane (MAM) protein required for hypoxia-induced mitochondrial fission and mitophagy. Autophagy 12: 1675-1676, 2016

27. Twig G, Elorza A, Molina AJ, Mohamed H, Wikstrom JD, Walzer G, Stiles L, Haigh SE, Katz S, Las G, et al: Fission and selective fusion govern mitochondrial segregation and elimination by autophagy. EMBO J 27: 433-446, 2008.

28. Kumar V and Maity S: ER Stress-sensor proteins and ER-mitochondrial crosstalk-signaling beyond (ER) stress response. Biomolecules 11: 173, 2021.

29. Szabadkai G, Simoni AM, Chami M, Wieckowski MR, Youle RJ and Rizzuto R: Drp-1-dependent division of the mitochondrial network blocks intraorganellar $\mathrm{Ca}^{2+}$ waves and protects against $\mathrm{Ca}^{2+}$-mediated apoptosis. Mol Cell 16: 59-68, 2004. 
30. Zhang J and Ney PA: Reticulocyte mitophagy: Monitoring mitochondrial clearance in a mammalian model. Autophagy 6: 405-408, 2010

31. Palikaras K, Lionaki E and Tavernarakis N: Mechanisms of mitophagy in cellular homeostasis, physiology and pathology. Nat Cell Biol 20: 1013-1022, 2018.

32. $\mathrm{Xu} \mathrm{Y,} \mathrm{Shen} \mathrm{J} \mathrm{and} \mathrm{Ran} \mathrm{Z:} \mathrm{Emerging} \mathrm{views} \mathrm{of} \mathrm{mitophagy} \mathrm{in}$ immunity and autoimmune diseases. Autophagy 16: 3-17, 2020.

33. Xu HM and Hu F: The role of autophagy and mitophagy in cancers. Arch Physiol Biochem: Oct 9, 2019 (Epub ahead of print). doi: 10.1080/13813455.2019.1675714.

34. Shida M, Kitajima Y, Nakamura J, Yanagihara K, Baba K, Wakiyama $\mathrm{K}$ and Noshiro $\mathrm{H}$ : Impaired mitophagy activates $\mathrm{mtROS} / \mathrm{HIF}-1 \alpha$ interplay and increases cancer aggressiveness in gastric cancer cells under hypoxia. Int J Oncol 48: 1379-1390, 2016.

35. Kim TW, Lee SJ, Park YJ, Park SY, Oh BM, Park YS, Kim BY, Lee YH, Cho HJ, Yoon SR, et al: Opa-interacting protein 5 modulates docetaxel-induced cell death via regulation of mitophagy in gastric cancer. Tumour Biol: Oct 15, 2017 (Epub ahead of print).

36. Marzetti E, Lorenzi M, Landi F, Picca A, Rosa F, Tanganelli F, Galli M, Doglietto GB, Pacelli F, Cesari M, et al: Altered mitochondrial quality control signaling in muscle of old gastric cancer patients with cachexia. Exp Gerontol 87: 92-99, 2017.

37. Yan H, Qiu C, Sun W, Gu M, Xiao F, Zou J and Zhang L: Yap regulates gastric cancer survival and migration via SIRT1/Mfn2/ mitophagy. Oncol Rep 39: 1671-1681, 2018.

38. Ferro F, Servais S, Besson P, Roger S, Dumas JF and Brisson L: Autophagy and mitophagy in cancer metabolic remodelling. Semin Cell Dev Biol 98: 129-138, 2020.

39. de Brito OM and Scorrano L: An intimate liaison: Spatial organization of the endoplasmic reticulum-mitochondria relationship. EMBO J 29: 2715-2723, 2010.

40. Santel A, Frank S, Gaume B, Herrler M, Youle RJ and Fuller MT: Mitofusin-1 protein is a generally expressed mediator of mitochondrial fusion in mammalian cells. J Cell Sci 116: 2763-2774, 2003.

41. Yu R,Liu T, Ning C, Tan F, Jin SB, Lendahl U, Zhao J and Nistér M The phosphorylation status of Ser-637 in dynamin-related protein 1 (Drp1) does not determine Drp1 recruitment to mitochondria. J Biol Chem 294: 17262-17277, 2019.

42. Amati-Bonneau P, Valentino ML, Reynier P, Gallardo ME, Bornstein B, Boissière A, Campos Y, Rivera $\mathrm{H}$, de la Aleja JG, Carroccia R, et al: OPA1 mutations induce mitochondrial DNA instability and optic atrophy 'plus' phenotypes. Brain 131: 338-351, 2008

43. Elezaby A, Sverdlov AL, Tu VH, Soni K, Luptak I, Qin F, Liesa M, Shirihai OS, Rimer J, Schaffer JE, et al: Mitochondrial remodeling in mice with cardiomyocyte-specific lipid overload. J Mol Cell Cardiol 79: 275-283, 2015.

44. Yao CH, Wang R, Wang Y, Kung CP, Weber JD and Patti GJ: Mitochondrial fusion supports increased oxidative phosphorylation during cell proliferation. Elife 8: e41351, 2019.

45. Rodríguez-Nuevo A, Díaz-Ramos A, Noguera E, Díaz-Sáez F, Duran X, Muñoz JP, Romero M, Plana N, Sebastián D, Tezze C, et al: Mitochondrial DNA and TLR9 drive muscle inflammation upon Opal deficiency. EMBO J 37: e96553, 2018.

46. Yang $\mathrm{H}$ and Zhang Z: Sepsis-induced myocardial dysfunction: the role of mitochondrial dysfunction. Inflamm Res: Mar 8, 2021 (Epub ahead of print). doi: 10.1007/s00011-021-01447-0.

47. Liesa M, Borda-d'Agua B, Medina-Gómez G, Lelliott CJ, Paz JC, Rojo M, Palacín M, Vidal-Puig A and Zorzano A: Mitochondrial fusion is increased by the nuclear coactivator PGC-1beta. PLoS One 3: e3613, 2008

48. Zhang GE, Jin HL, Lin XK, Chen C, Liu XS, Zhang Q and Yu JR: Anti-tumor effects of Mfn2 in gastric cancer. Int J Mol Sci 14: 13005-13021, 2013.

49. Tang BL: Sirt1 and the mitochondria. Mol Cells 39: 87-95, 2016.

50. Li H, He C, Wang X, Wang H, Nan G and Fang L: MicroRNA-183 affects the development of gastric cancer by regulating autophagy via MALAT1-miR-183-SIRT1 axis and PI3K/AKT/ mTOR signals. Artif Cells Nanomed Biotechnol 47: 3163-3171, 2019.

51. An Y, Wang B, Wang X, Dong G, Jia J and Yang Q: SIRT1 inhibits chemoresistance and cancer stemness of gastric cancer by initiating an AMPK/FOXO3 positive feedback loop. Cell Death Dis 11: 115,2020.

52. Gowans GJ and Hardie DG: AMPK: A cellular energy sensor primarily regulated by AMP. Biochem Soc Trans 42: 71-75, 2014
53. Shi HJ, Xu C, Liu MY, Wang BK, Liu WB, Chen DH, Zhang L, $\mathrm{Xu} \mathrm{CY}$ and Li XF: Resveratrol improves the energy sensing and glycolipid metabolism of blunt snout bream megalobrama amblycephala fed high-carbohydrate diets by activating the AMPK-SIRT1-PGC-1 $\alpha$ network. Front Physiol 9: 1258, 2018.

54. Scarpulla RC: Metabolic control of mitochondrial biogenesis through the PGC-1 family regulatory network. Biochim Biophys Acta 1813: 1269-1278, 2011

55. Yang H, Du L and Zhang Z: Potential biomarkers in septic shock besides lactate. Exp Biol Med (Maywood) 245: 1066-1072, 2020

56. Bose S and Le A: Glucose metabolism in cancer. Adv Exp Med Biol 1063: 3-12, 2018.

57. Pavlova NN and Thompson CB: The emerging hallmarks of cancer metabolism. Cell Metab 23: 27-47, 2016.

58. Alzahrani AS: PI3K/Akt/mTOR inhibitors in cancer: At the bench and bedside. Semin Cancer Biol 59: 125-132, 2019.

59. Rodríguez-Enríquez S, Marín-Hernández Á, GallardoPérez JC, et al: Transcriptional regulation of energy metabolism in cancer cells. Cells 8: 1225, 2019.

60. Gonnella R, Santarelli R, Farina A, Granato M, D'Orazi G, Faggioni $\mathrm{A}$ and Cirone $\mathrm{M}$ : Kaposi sarcoma associated herpesvirus (KSHV) induces AKT hyperphosphorylation, bortezomib-resistance and GLUT-1 plasma membrane exposure in THP-1 monocytic cell line. J Exp Clin Cancer Res 32: 79, 2013.

61. Semenza GL: Targeting HIF-1 for cancer therapy. Nat Rev Cancer 3: 721-732, 2003.

62. Gottlieb E and Tomlinson IP: Mitochondrial tumour suppressors: A genetic and biochemical update. Nat Rev Cancer 5: 857-866, 2005.

63. Koch A, Ebert EV, Seitz T, Dietrich P, Berneburg M, Bosserhoff A and Hellerbrand C: Characterization of glycolysis-related gene expression in malignant melanoma. Pathol Res Pract 216: 152752,2020

64. Hägg M and Wennström S: Activation of hypoxia-induced transcription in normoxia. Exp Cell Res 306: 180-191, 2005.

65. Gogvadze V, Orrenius S and Zhivotovsky B: Mitochondria in cancer cells: What is so special about them? Trends Cell Biol 18: 165-173, 2008.

66. Zhou Y, Xu Q, Shang J, Lu L and Chen G: Crocin inhibits the migration, invasion, and epithelial-mesenchymal transition of gastric cancer cells via miR-320/KLF5/HIF-1 $\alpha$ signaling. J Cell Physiol 234: 17876-17885, 2019.

67. Gan L, Meng J, Xu M, Liu M, Qi Y, Tan C, Wang Y, Zhang P, Weng W, Sheng W, et al: Extracellular matrix protein 1 promotes cell metastasis and glucose metabolism by inducing integrin $\beta 4 / \mathrm{FAK} / \mathrm{SOX} 2 / \mathrm{HIF}-1 \alpha$ signaling pathway in gastric cancer. Oncogene 37: 744-755, 2018.

68. Oya Y, Hayakawa Y and Koike K: Tumor microenvironment in gastric cancers. Cancer Sci 111: 2696-2707, 2020.

69. Bruning U, Fitzpatrick SF, Frank T, Birtwistle M, Taylor CT and Cheong A: NFKB and HIF display synergistic behaviour during hypoxic inflammation. Cell Mol Life Sci 69: 1319-1329, 2012

70. D'Ignazio L, Bandarra D and Rocha S: NF- $\kappa$ B and HIF crosstalk in immune responses. FEBS J 283: 413-424, 2016.

71. Lavecchia A, Di Giovanni C and Cerchia C: Novel inhibitors of signal transducer and activator of transcription 3 signaling pathway: An update on the recent patent literature. Expert Opin Ther Pat 24: 383-400, 2014.

72. Mishra P and Chan DC: Metabolic regulation of mitochondrial dynamics. J Cell Biol 212: 379-387, 2016.

73. Wang $\mathrm{HH}, \mathrm{Wu} \mathrm{YJ}$, Tseng YM, Su CH, Hsieh CL and Yeh HI: Mitochondrial fission protein 1 up-regulation ameliorates senescence-related endothelial dysfunction of human endothelial progenitor cells. Angiogenesis 22: 569-582, 2019.

74. Liang J, Yang Y, Bai L, Li F and Li E: DRP1 upregulation promotes pancreatic cancer growth and metastasis through increased aerobic glycolysis. J Gastroenterol Hepatol 35: 885-895, 2020.

75. Almeida L, Lochner M, Berod L and Sparwasser T: Metabolic pathways in $\mathrm{T}$ cell activation and lineage differentiation. Semin Immunol 28: 514-524, 2016

76. Van Wyngene L, Vandewalle J and Libert C: Reprogramming of basic metabolic pathways in microbial sepsis: therapeutic targets at last? EMBO Mol Med 10: e8712, 2018.

77. Ren R, Guo J, Shi J, Tian Y, Li M and Kang H: PKM2 regulates angiogenesis of VR-EPCs through modulating glycolysis, mitochondrial fission, and fusion. J Cell Physiol 235: 6204-6217, 2020.

78. Seo BJ, Choi J, La H, Habib O, Choi Y, Hong K and Do JT: Role of mitochondrial fission-related genes in mitochondrial morphology and energy metabolism in mouse embryonic stem cells. Redox Biol 36: 101599, 2020. 
79. Hsu PP and Sabatini DM: Cancer cell metabolism: Warburg and beyond. Cell 134: 703-707, 2008.

80. Lin XH, Qiu BQ, Ma M, Zhang R, Hsu SJ, Liu HH, Chen J, Gao DM, Cui JF, Ren ZG, et al: Suppressing DRP1-mediated mitochondrial fission and mitophagy increases mitochondrial apoptosis of hepatocellular carcinoma cells in the setting of hypoxia. Oncogenesis 9: 67, 2020.

81. Liu B, Fan Y, Song Z, Han B, Meng Y, Cao P and Tan K: Identification of DRP1 as a prognostic factor correlated with immune infiltration in breast cancer. Int Immunopharmacol 89: 107078, 2020.

82. Rehman J, Zhang HJ, Toth PT, Zhang Y, Marsboom G, Hong Z, Salgia R, Husain AN, Wietholt C and Archer SL: Inhibition of mitochondrial fission prevents cell cycle progression in lung cancer. FASEB J 26: 2175-2186, 2012.

83. Gao T, Zhang X, Zhao J, Zhou F, Wang Y, Zhao Z, Xing J, Chen B, Li J and Liu S: SIK2 promotes reprogramming of glucose metabolism through PI3K/AKT/HIF-1 $\alpha$ pathway and Drp1-mediated mitochondrial fission in ovarian cancer. Cancer Lett 469: 89-101, 2020.
84. Nagdas S, Kashatus JA, Nascimento A, Hussain SS, Trainor RE, Pollock SR, Adair SJ, Michaels AD, Sesaki H, Stelow EB, et al: Drp1 promotes KRas-driven metabolic changes to drive pancreatic tumor growth. Cell Rep 28: 1845-1859.e5, 2019.

85. Bray F, Ferlay J, Soerjomataram I, Siegel RL, Torre LA and Jemal A: Global cancer statistics 2018: GLOBOCAN estimates of incidence and mortality worldwide for 36 cancers in 185 countries. CA Cancer J Clin 68: 394-424, 2018.

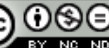

This work is licensed under a Creative Commons Attribution-NonCommercial-NoDerivatives 4.0 International (CC BY-NC-ND 4.0) License. 